\title{
Arbitrarily Shaped Objects Relighting Using an RGB-D Camera
}

\author{
Takuya Ikeda*, Francois de Sorbier* and Hideo Saito* \\ ${ }^{*}$ Graduate School of Science and Technology \\ Keio university, Japan \\ Email: \{ikeda,fdesorbi,saito\}@hvrl.ics.keio.ac.jp
}

\begin{abstract}
Usually, relighting techniques require knowledge about the shape of the target object and the lighting environment. The quality of the result is highly dependent on the normals of the object because they are used in the computation of the illumination. In this paper, we propose a new relighting approach for arbitrarily shaped objects using an RGB-D camera such as the Microsoft's Kinect. The depth map is useful to estimate the normals of the object, but can be inaccurate because of the noise such as discrete depth values or missing data. An accurate segmentation of the target region for relighting is also an open issue since the boundaries in the depth map does not always match color's ones. We focus on the depth map modification to segment the object region and normal estimation for accurate relighting. In our experiments, we adapted some normal estimation methods from modified depth map and evaluated the accuracy of the relighting results. We discuss the effectiveness of relighting approach for an arbitrarily shaped object and the possibility of a real time relighting.
\end{abstract}

\section{INTRODUCTION}

Relighting is a currently active research topic in computer vision. It can be applied not only to videos but also to matching technique that use SIFT or SURF which are weak on change of illumination conditions. However, getting an accurate relighting result remains a complicated task. Generally, relighting technique needs the object shape, the reflectance property and light environments. The accuracy of the results depends on accuracy of each of these factors.

In previous works, there are some researches focusing on relighting for a fixed object because of the difficulty of obtaining the exact shape and the reflectance property in real time. Debevec proposed to acquire the reflectance field of the object by using Light Stage [1]. They captured the object under 2048 light directions for estimating the reflectance functions of the object, so that the high accuracy relighting results can be obtained by using these functions. This method doesn't need to estimate the object normal because the reflectance functions include not only the object's diffuse and specular reflectance but also visibility from all light directions. In contrast, we propose the relighting system without such a lot of images. Zhen et al. [2] and Oswald et al. [3] proposed relighting techniques applied on human face images by using compute vision techniques. The human face geometry can be estimated with a morphable model of 3D faces. They obtained the normal map by using this technique.

Zhen et al. proposed a rate image technique for relighting. Processing time of this method is fast because they only considered the diffuse component and approximated the radiance environment map with Spherical Harmonics (SH) [4]. In our case, we also use this technique in our proposed relighting flow. Oswald et al. also estimated the face geometry and relighted it by considering not only the diffuse component but also the specular one. However, as mentioned before, these works treated a stationary object or one image.

In this paper, we proposed arbitrarily shaped objects relighting using an RGB-D camera. Depth sensor can obtain a scene geometry in real time (more than $30 \mathrm{fps}$ ). As far as we have investigated, such approaches using RGB-D camera have not been proposed yet. As a first step, we relight arbitrarily shaped objects from a single color image and depth map. For arranging the object on to another scene background, segmentation of the correct object region is important. And, the object normals significantly affect the accuracy of the relighting result. However, the boundaries in the depth map does not always match color's ones and the depth map has noisy data such as discrete depth values or missing data. Because of these, it is not easy to accurately segment and to estimate the normals from a single depth map. We focus on these two processes. Firstly, we modify the depth map using color and the depth information. Thus, we obtain improved depth map especially in the boundary between the object and background and the object region is segmented with a simple depth thresholding. After that, we estimate the normal map from the modified depth map. Radu et al. proposed normal estimation based on $k$ nearest neighbor [5] and Stefan et al. also proposed methods using integral images [6]. In our experiments, we compare with five normal estimation methods including the above.

In our flow, we use some processing which are integral image, kmeans clustering and $\mathrm{SH}$ rotation. Recently, parallel algorithms of these processing are proposed [7], [8], [9]. They are available for a real time relighting. In Sec.V, we discuss about the possibility of a real time relighting.

\section{RELIGHTING THEORY}

\section{A. Spherical Harmonics Lighting}

In this section, we explain about a relighting theory. Image based lighting is very useful for rendering synthetic objects into real scenes such as in augmented reality. This technique is also useful for the relighting. We assumed that a light source come from infinity distance. For considering a point light source in all directions, we assume that light environment is distributed on a unit sphere. The irradiance $E(\boldsymbol{x})$ observed at a point $\boldsymbol{x}=(x, y, z)$ is given by an integral over the sphere 
$\Omega$.

$$
E(\boldsymbol{x})=\int_{\Omega} L(\omega) \max ((\omega \cdot \boldsymbol{n}), 0) d \omega
$$

Note that $L(\omega)$ represents the light intensity along the direction vector $\omega \cdot(\omega \cdot \boldsymbol{n})$ is the cosine term and $\boldsymbol{n}$ is a normal vector at a point $\boldsymbol{x}$. This model considers only shading model (does not consider shadow).

We obtain a light environment from captured light probe [10] and approximate the light environment in SH [4]. Using the notation of [4], the irradiance of point $x$ can be represented as a linear combination of SH basis functions,

$$
E(\boldsymbol{x})=\sum_{l=0}^{\infty} \sum_{m=-l}^{l} A_{l}(\theta) L_{l m} Y_{l m}(\omega)
$$

For computing $E(\boldsymbol{x})$ with arbitrary normal vector $\boldsymbol{n}$, we compute the rotation of the $\mathrm{SH}$ [7] for a term of $A_{l}(\theta)$. Preliminarily, we compute the standard cosine term $A_{l}^{\text {std }}$ is equal to $A_{l}(0)$. After normal estimation, we compute $\mathrm{SH}$ rotation from $A_{l}^{\text {std }}$ to $A_{l}(\theta)$. Note that, $\theta$ is a elevation angle corresponding to the normal direction $\boldsymbol{n}$.

Ramamoorthi and Hanrahan [4] showed that for diffuse reflectance, only 9 coefficients are needed to approximate the irradiance function. Assuming Lambertian surface reflectance, the pixel value of $i(\boldsymbol{u})$ is written as

$$
i(\boldsymbol{u})=R_{d} \sum_{l=0}^{2} \sum_{m=-l}^{l} A_{l}(\theta) L_{l m} Y_{l m}(\omega)
$$

where $\boldsymbol{u}=(u, v)$ represents pixel position corresponding to point $x$. $R_{d}$ is a diffuse reflection parameter of the object surface. Since lambertian surface uniformly reflects the light to all directions, $R_{d}$ is set to a constant value.

\section{B. Relighting in different light environment}

In relighting part, we adapt the rate image technique [2]. We need to know two light environments. The first is a scene captured the object and the other is a scene which light environment is different from the first one. In this paper, we call these two scenes $S r c$ and Dst respectively. If $S r c$ and $D s t$ light environments and the object normal are known, the pixel intensity of relighting image is obtained by the ratio of Eq. (2),

$$
i^{d s t}(\boldsymbol{u})=i^{s r c}(\boldsymbol{u}) \frac{E^{d s t}(\boldsymbol{x})}{E^{s r c}(\boldsymbol{x})}
$$

Note that $i^{s r c}(\boldsymbol{u})$ is an input pixel value and $E^{s r c}$ is calculated from the $S r c$ light environment, $i^{d s t}(\boldsymbol{u})$ and $E^{d s t}$ are same representation in the Dst light environment. By computing the rate of two irradiance functions, the constant value $R_{d}$ can be removed. This means that we can obtain the religthing results without estimating the reflectance property.

\section{RELIGHTING USING AN RGB-D CAMERA}

In this section, we explain about the proposed relighting approach. Our relighting flow is shown in Fig. 1. Input images are $S r c$ and Dst light environments, the object color image, depth map and Dst background image. Light environments are represented in cube map. For relighting, segmentation of the object region and normal estimation is very important. In our strategy, the object region is segmented using a depth thresholding. However, the boundaries in depth map does not always match color's ones because of the noisy depth map. Therefore, before segmentation, we modify the depth map around the object boundary. After that, normal map is estimated using the modified depth map and we compute the SH rotation for the term of $A_{l}(\theta)$. Finally, the relighting result is obtained by computing Eq. (4) and it is arranged on Dst background.

The quality of relighting result depends on the normal map accuracy. There are many methods to estimate the normal from the depth information. In Sec.V, we apply five normal estimation methods and evaluate the accuracy of results. Following subsection, we explain about how to modify depth map for segmentation and five normal estimation methods.

\section{A. Depth Map Modification for Exact Segmentation}

The strategy of depth map modification is similar to the one from Chen et al. [11]. In our case, we only modify the boundary between the object and background. In the first step, we set the invalid depth region around the object boundary. In the second step, the invalid depth is filled by using color and depth information of the neighbor pixels, as depicted in Fig. 2.

Firstly, we segment the object region by using simple depth thresholding. Large segmented region which is shown in Fig. 2 (a) is obtained by applying a dilatation operator. After that, we compute the invalid depth region by using color and depth edge information. Both image edges are obtained by Canny Operator. The depth edge region mask $M_{d e r}$ is obtained by expanding the edge pixels with a square window. Same operation is adapted in the color image and we obtain the color edge region mask $M_{c e r}$. Then, final mask of the invalid depth region $M_{i d r}$ is obtained as follows:

$$
M_{i d r}=M_{d e r} A N D M_{c e r}
$$

The color edge includes not only the object boundary but also texture edge in the object or background. Compared with the color edge, most of the depth edge is detected near the object boundary because the distance between the object boundary and background is large. The window size of depth edge should be larger than color one. By doing this, $M_{i d r}$ shown in Fig. 2 (b) includes around the boundary in color image. Note that the mask region of $M_{i d r}$ is replaced color information. Invalid depth map $D_{i}$ is obtained using the raw depth map with $M_{i d r}$.

The second step is filling the invalid depth. The invalid depth filling equation is the same as [11]. The estimated depth value $D_{i}(\boldsymbol{u})$ of invalid pixel $\boldsymbol{u}$ is calculated as follows:

$D_{i}^{t}(\boldsymbol{u})=\frac{1}{k(\boldsymbol{u})} \sum_{\boldsymbol{v} \in \Omega_{s}, D_{i}^{t-1} \notin 0} g_{s}(\boldsymbol{u}-\boldsymbol{v}) g_{c}(i(\boldsymbol{u})-i(\boldsymbol{v})) D_{i}^{t-1}(\boldsymbol{v})$

where $g_{s}$ is spatial weight and $g_{c}$ is the color similarity weight. They are Gaussian function and $\sigma_{s}, \sigma_{c}$ are standard deviation respectively. $\Omega_{s}$ is a square window around an invalid pixel $u$ and $\left.k_{(} \boldsymbol{u}\right)$ is a normalizing factor. $D_{i}(\boldsymbol{v})$ is a valid depth value of pixel $\boldsymbol{v} \cdot \boldsymbol{u}-\boldsymbol{v}$ and $i(\boldsymbol{u})-i(\boldsymbol{v})$ represent Euclidean distance in image space and color space respectively. $D_{i}^{t}$ is obtained 


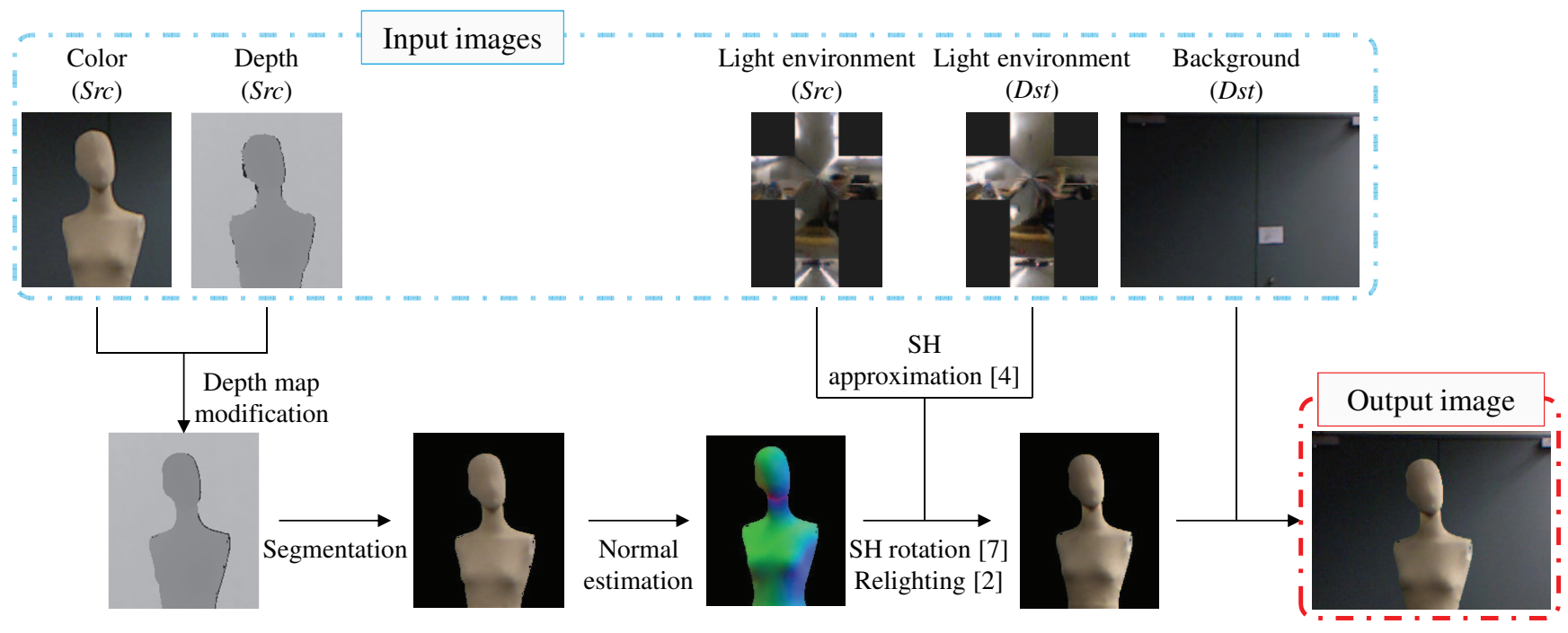

Fig. 1. Flow of proposed our relighting.

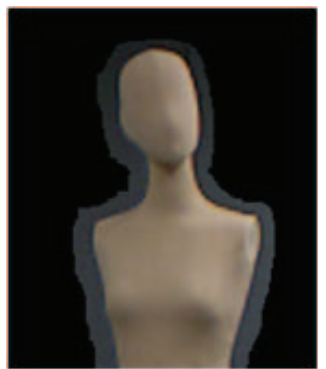

(a)

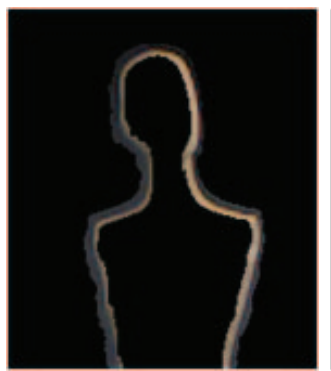

(b)

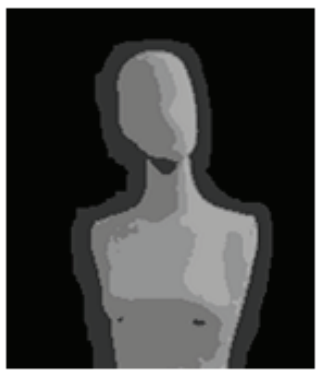

(c)

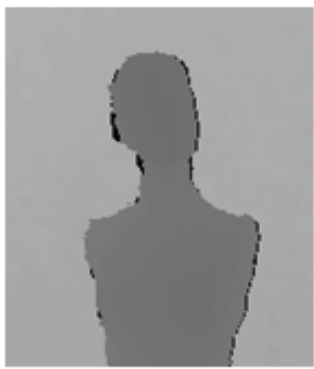

(d)

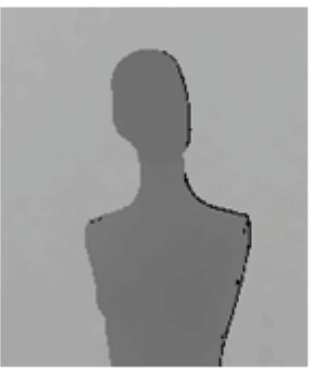

(e)

Fig. 2. Depth modification. (a)large segmented image, (b) depth image, (c) kmeans clustering image, (d) invalid depth mask with color information, (e) depth modified image.

by computing Eq. (6) $t$ times. By repeating this process with updating $D_{i}$, we obtain the modified depth map $D_{m}$.

In order to obtain better segmentation results, the depth value from an invalid pixel in the object region should be estimated using the valid depth value in the same region. To satisfy this situation, we compute color segmented image using Kmeans clustering that is shown in Fig. 2 (c). After that, each pixel in the color segmented result has a label. The depth value of $\boldsymbol{u}$ is estimated using neighbor pixels which label is same with $\boldsymbol{u}$. The depth modified result is shown in Fig. 2 (e). Compared with raw depth map shown in Fig. 2 (d), the object boundary is improved by using this process. However, sometimes Kmeans clustering result has minor label pixel. It means that the label of interest pixel and neighbor pixels are different. In such a situation, if the minor label pixel is invalid depth, our method cannot estimate its depth value. This behavior depends on kmeans cluster number and iteration number. In our experiments, we have set the appropriate value for each parameter. After depth modification, we resegment the object region using modified depth map.

\section{B. Normal Estimation from Modified Depth Map}

After depth map modification and segmentation, we obtain ideal depth map in the object region. When depth map and the camera's intrinsic calibration parameter are known, it can be converted into 3D vertex map $\boldsymbol{V}$ in the camera's coordinate space. After that, we can estimate the normal map from $V$. In this section, we explain about five normal estimation methods.

The first one is a very simple approach. Given a vertex map $V$, a normal vector of pixel $\boldsymbol{u}$ is computed by using adjacent pixel vertices as follows: $\boldsymbol{n}(\boldsymbol{u})=(V(u+1, v)-V(u, v)) \times$ $(V(u, v+1)-V(u, v))$ after normalization, we get normal map $N=\boldsymbol{n} /\|\boldsymbol{n}\|$.

However, the depth values are discrete and contain noise. To reduce them and obtain smoothing depth map, we apply a bilateral filter [12] to the raw depth map before computing vertex map. The new depth map with reduced noise $D_{b}$ as follows:

$D_{b}(\boldsymbol{u})=\frac{1}{k^{\prime}(\boldsymbol{u})} \sum_{\boldsymbol{v} \in \Omega_{s^{\prime}}} g_{s^{\prime}}(\boldsymbol{u}-\boldsymbol{v}) g_{d}\left(D_{m}(\boldsymbol{u})-D_{m}(\boldsymbol{v})\right) D_{m}(\boldsymbol{v})$

$\left.k^{\prime} \boldsymbol{u}\right)$ are normalizing factor, and $g_{d}$ is the depth similarity weight. $g_{s^{\prime}}$ and $g_{d}$ are Gaussian function with $\sigma_{s^{\prime}}$ and $\sigma_{d}$ respectively. $\Omega_{s^{\prime}}$ needs appropriate value for smoothing. If $\Omega_{s^{\prime}}$ is too large, very smoothed depth map is obtained and the detail of normal information is lost. In contrast, if $\Omega_{s^{\prime}}$ is too small, the normal map is roughness shape. After filtering 
with the appropriate window size, normal map is estimated by performing the same process as the first method.

These two methods are simple normal estimations. Other three methods are novel approaches proposed by Radu et al. [5] and Stefan et al. [6]. Radu et al. estimated the normal vector using $k$ nearest neighbor $(\mathrm{kNN})$ based approach which is described in [5]. To apply this method, vertex map $\boldsymbol{V}$ needs to be integrated into a $\mathrm{kD}$-tree. When the query point is given, $k$ neighbor points are searched and the normal vector is estimated by performing a Principal Component Analysis on the neighborhood's covariance matrix. Stefan et al. proposed two normal estimation methods using integral images which are also described in [6]. The first is based on conventional matrix $(\mathrm{CM})$. It creates nine integral images to compute the normal for specific point from the covariance matrix of its local neighborhood. The other method based on smoothed depth changes (SDC). It also computes six integral images and smoothing area map for estimating normal. In these two methods, the input vertex form is same as the first and second methods. Computation times of them are faster than [5] because of vertex data doesn't need to change its form. However, this method cannot estimate the normal around the boundary if it has a large difference of depth value. Therefore, we replace the invalid normal with the results of second method. We use bilateral filtered depth map $D_{b}(\boldsymbol{u})$ as an input in these three methods. In our experiments, we call all methods as RAW, BILATERAL, kNN, CM+BILATERAL and SDC+BILATERAL respectively.

\section{IMPLEMENTATION}

In our experiments, the RGB-D camera was the Microsoft's Kinect. All the input data reported in this paper are captured by it. Before the relighting, we captured the Src and Dst light probes [10] and Dst background. Also, we recorded the color and depth information over 10 seconds. After that, we relighted the three hundred frames. We used two objects of different shape Mannequin and Duck. The first type is human shape, the other is toy type includes specular reflectance. Background images and light environments are shown in Fig. 3. In the experiment of Mannequin, Src environment is Room1 and Dst one is Room2. As well as Mannequin, Src environment is Room 3 and Dst one is Room4 in the experiment of Duck. The objects are fixed while capturing $S r c$ and Dst environments for evaluating Ground Truth. Implementation parameters of Mannequin are shown in Table.I. The resolution of input is $640 \times 480$ image, and all processes are computed on a PC with Intel Core i7-3940XM 3.00GHz and $32.0 \mathrm{~GB}$ memory. In the next section, we discuss the relighting results and processing time.

\section{EXPERIMENTAL RESULTS}

Fig. 5 and Fig. 6 show the normal map represented in color space and the relighting results. The upper side of first column is input image and the lower side is Ground Truth, from second to last columns show normal map and relighting results.

First we discuss about the results of Mannequin. In the light environment of Room1 shown in Fig. 3 (a), two fluorescent lights are placed the left and right sides of the ceiling. Therefore, the object shading shown in the upner side

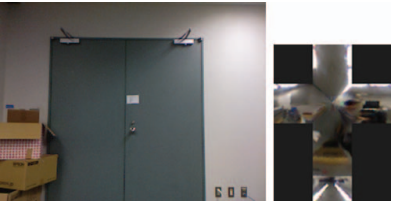

(a)

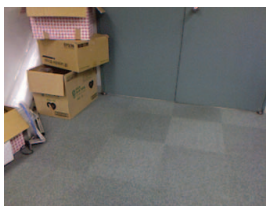

(c)

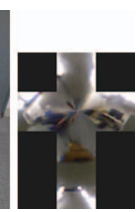

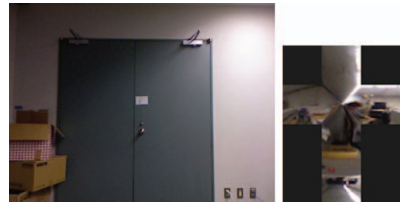

(b)

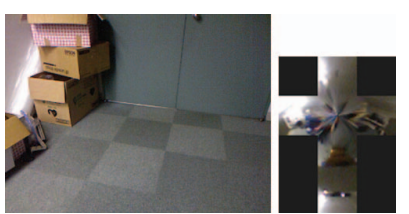

(d)
Fig. 3. Background images and light environments using our experiments (a) Room1, (b) Room2, (c) Room3, (d) Room4. For evaluation, Room1 and Room 2 are captured with same camera pose and position but light environments are different. Room 3 and Room 4 are captured another camera pose and position under two different light conditions.

TABle I. Parameters about experiment of Mannequin

\begin{tabular}{|l|c|c|}
\hline Processing & parameter & value \\
\hline \hline Invalid depth mask & Window size of $M_{\text {der }}$ & $10 \times 10$ \\
\cline { 2 - 3 } & Window size of $M_{c e r}$ & $6 \times 6$ \\
\hline Invalid depth filling & $\sigma_{s}$ & 1.4 \\
\cline { 2 - 3 } & $\sigma_{c}$ & 1.2 \\
\cline { 2 - 3 } & $\Omega_{s}$ & $6 \times 6$ \\
\cline { 2 - 3 } & $t$ & 40 \\
\hline Kmeans clustering & $k$ & 10 \\
\cline { 2 - 3 } & Iteration number & 10 \\
\hline Bilateral filter & $\sigma_{s^{\prime}}$ & 150 \\
\cline { 2 - 3 } & $\sigma_{d}$ & 60 \\
\cline { 2 - 3 } & $\Omega_{s^{\prime}}$ & $9 \times 9$ \\
\hline
\end{tabular}

of Fig. 5 (a) is bright overall without the shadow observed in the neck and under the chest. On the other hand, in Room2 shown in Fig. 3 (b), one fluorescent light is placed the right side of the ceiling. The object captured in Room 2 is shown in the lower side of Fig. 5 (a). The right side of the object is bright and the left side is dark. The goal of this experiment is that to create a image same as Ground Truth by relighting the input image. The columns (b)-(f) in Fig. 5 are normal maps arranged in the upper side and relighting results arranged in the lower side. The object region is exact segmented by using our proposed depth map modification. Thus, the object is naturally arranged on Dst background. All relighting results except for RAW are similar with Ground Truth. The result of RAW is observed dark or bright pixels partially and is unnatural image. The cause of this is noisy depth map. The raw depth map has discrete values and the vertex map in the object isn't smooth. Thus, normal direction becomes same in some regions. This is seen from that the same pixel value is observed partially in the normal map image. Compared with RAW, BILATERAL result are better smooth shading. After filtering process, the depth map is smoothed without lose of edge information. But Smoothness of the normal map is rough because of outliers of depth value. As we mentioned before, the raw depth map has noise such as outliers compared with neighbor pixel. In filtering process, such data is also included and thus the vertex map and normal map becomes rough. The relighting result is observed unnatural dark or bright shading in the part of body. $\mathrm{kNN}$ result seems to be the most similar with Ground Truth in all methods. $\mathrm{kNN}$ method discards the outliers when estimate 


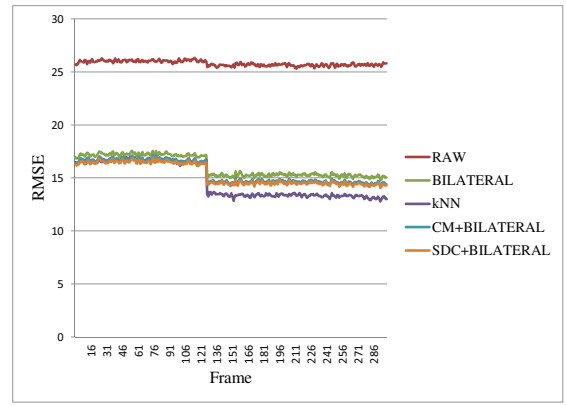

(a)

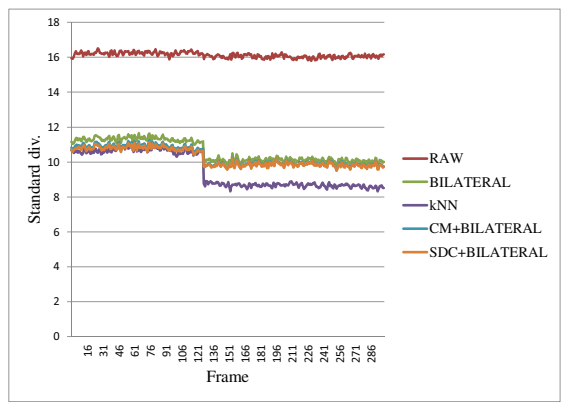

(b)

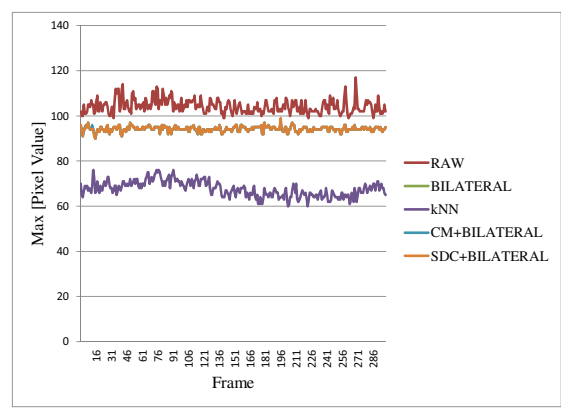

(c)

Fig. 4. Evaluation of Mannequin. (a) RMSE, (b) Std. dev., (c) Maximum (Max) value of the absolute difference between the results and Ground Truth.

the normal and thus the high accuracy result can be obtained. Relighting result isn't appeared unnatural shading like RAW and BILATERAL. On the other hand, CM+BILATERAL and SDC + BILATERAL seem to be almost same result and similar with Ground Truth. Compared with BILTERAL, normal maps and relighting results are smooth. using adjacent pixel vertices While the normal is estimated by using adjacent pixel vertices in BILATERAL, CM and SDC consider the neighbor pixel with changing the window size. Observing the results more carefully, normal maps of CM+BILATERAL and SDC + BILATERAL are slightly rough especially in hip and left shoulder. The window size is small around the object boundary because the window size is defined by considering depth changing. Therefore, such a roughness are observed in these results.

Next, we discuss about results of Duck shown in Fig. 6. Arrangement of images is same as Fig. 5. In case of Duck, we relighted the object shading from under the Room3 to Room4. These environments are shown in Fig. 3 (c) and (d). It seems to be almost the same quality as case of Mannequin. Differences of Mannequin are that Duck has a specular reflection notably and shadow is observed on the green table in Ground Truth. We only consider the diffuse component and thus these results do not take into account the specular reflection and shadow that seen in Ground Truth. To obtain more accurate results, we need to consider the visibility between the object and the light positions and to consider the specular reflection model. From our experiments, our method is useful for these simple object. If the target object shape is complex such as a tree, it is impossible to relight because obtaining its correct shape from Kinect is quite difficult.

Again, we discuss about evaluation of Mannequin. We relighted 300 images which are captured in advance and evaluated the relighting results of them. All result images are compared with Ground Truth. Quantitative evaluation of Mannequin is shown in Fig. 4. It shows the RMSE and Std. dev., Maximum (Max) value of the absolute difference between the results and Ground Truth. Result of RAW is the worst in all evaluation and this method may not be useful. BILATERAL, CM+BILATERAL and SDC+BILATERAL are quite same in Max and BILATERAL is slight worse than the rest two methods in RMSE and Std. dev.. This result is caused by the roughness of each normal map, that is mentioned above. $\mathrm{kNN}$ is the best accuracy, especially, Max is very low compared with other methods. It means that the relighting result and Ground Truth are the closest.

TABLE II. COMPUTATION TIME OF Mannequin

\begin{tabular}{|l|c|}
\hline Processing & time $(\mathrm{msec})$ \\
\hline \hline Depth map modification & 158.87 \\
\hline RAW & 10.87 \\
\hline BILATERAL & 393.9 \\
\hline kNN & 1409.46 \\
\hline CM+BILATERAL & 467.64 \\
\hline SDC+BILATERAL & 432.63 \\
\hline
\end{tabular}

Computation time of Mannequin is shown in Table.II. This table shows only depth map modification and normal estimation methods. Considering the accuracy and processing time, RAW and BILATERAL are smaller processing time but accuracy isn't better than the others. On the other hand, $\mathrm{kNN}$ takes a large time, but the accuracy is the best in all methods. CM+BILATERAL and SDC+BILATERAL take a time a little more than BILATERAL. However the relighting results are better. As we mentioned in Sec.I, parallel processing algorithms [7], [8], [9] are possible in these two methods and our relighitng flow, so that all processes can be implemented in GPU for faster processing time. So, it is possible to perform a real time relighting by accelerating the proposed method.

\section{CONCLUSION AND FUTURE WORK}

In this paper, we proposed the novel relighting method using an RGB-D camera. The difficulty of relighting from the raw depth map is mainly caused by depth data. To solve this problem, we proposed the depth map modification for exact segmentation and compared the five normal estimation methods from such a noisy depth data. To solve this problem, we proposed a method for the depth map modification for exact segmentation of the object region. We then compared five normal estimation methods from such a noisy depth data. In our experiments, we showed the relighting results for two objects of different shape. The object region were segmented exactly using our proposed method, so that we can obtain relighitng results similar with Ground Truth. We discussed the accuracy of them and computation time and mentioned about the possibility of expansion to a real time relighting.

\section{REFERENCES}

[1] Paul Debevec, Tim Hawkins, Chris Tchou and Haarm-Pieter Duiker, Westley Sarokin and Mark Sagar, "Acquiring the reflectance field of a human face", in SIGGRAPH, pp. 145-156, 2000.

[2] Zhen Wen, Zicheng Liu and Thomas S Huang, "Face Relighting with Radiance Environment Maps”, in CVPR, pp. 158-165, 2003. 

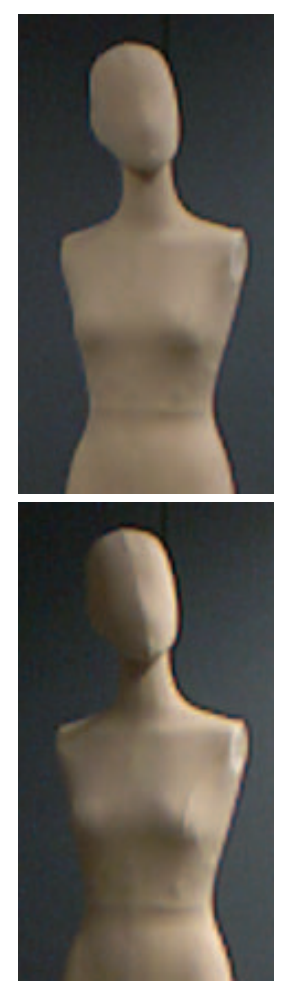

(a)
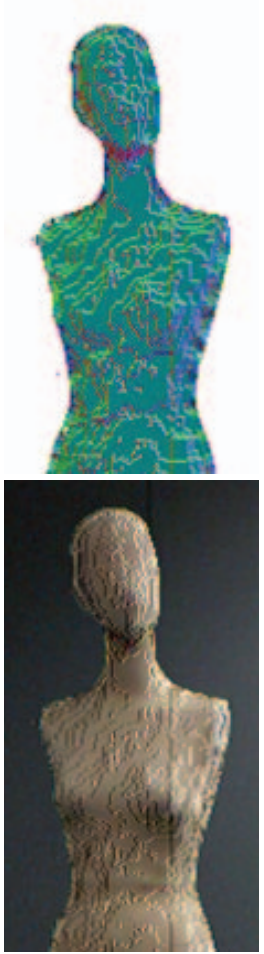

(b)
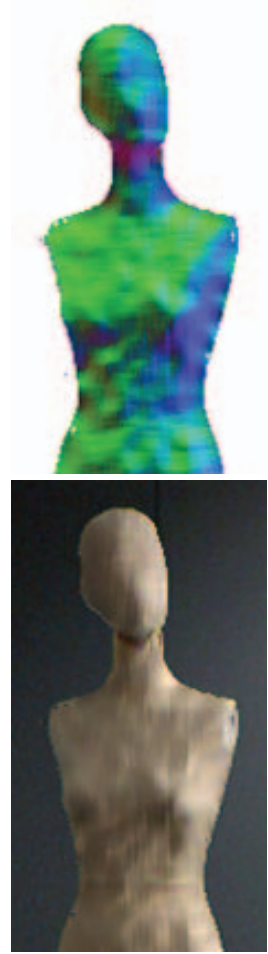

(c)

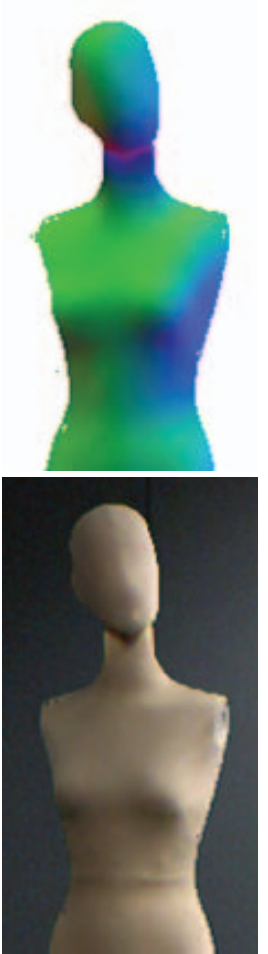

(d)
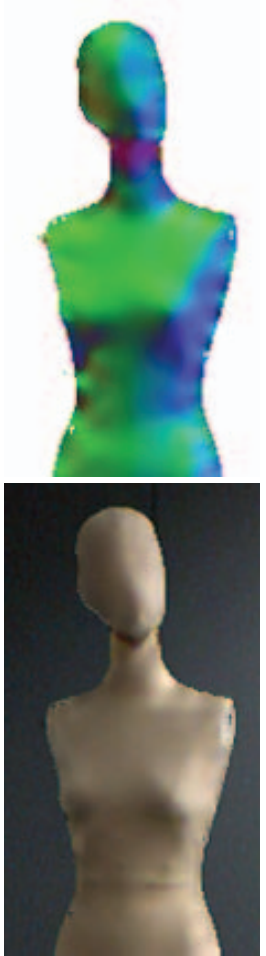

(e)

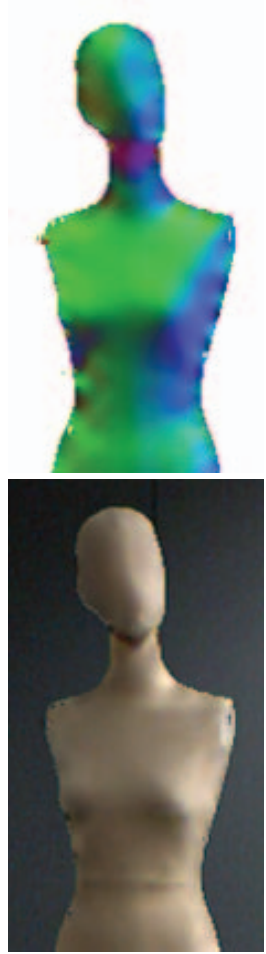

(f)

Fig. 5. Relighting results of Mannequin. Src environment is Room1, Dst environment is Room2. (a) the upper side is input color, the lower side is Ground Truth, the upper side of (b)-(f) are normal map and the lower side are relighting results. Normal map is represented in color space. Columns (b)-(f) are RAW, BILATERAL, kNN, CM+BILATERAL, SDC+BILATERAL respectively.
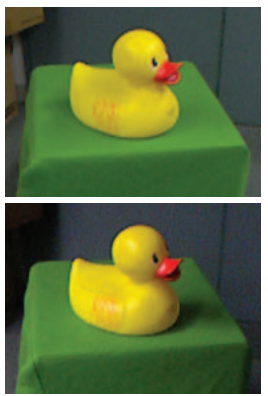

(a)
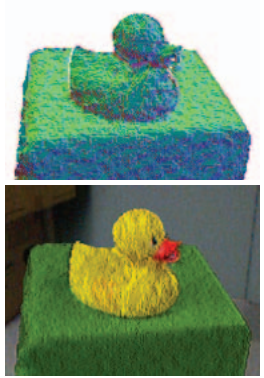

(b)
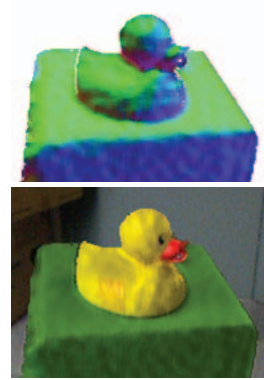

(c)
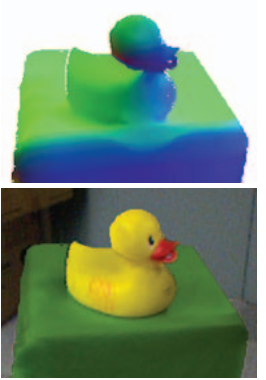

(d)
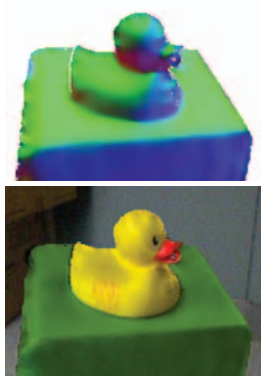

(e)

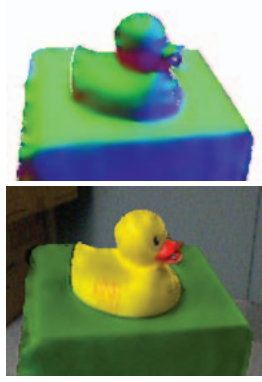

(f)

Fig. 6. Relighting results of Duck. Arrangement of images is same as Fig. 5. Src environment is Room3, Dst environment is Room4

[3] Oswald Aldrian and William A. P. Smith, "Inverse rendering with a morphable model: A multilinear approach", in BMVA, pp. 88.1-88.10, 2011

[4] Ravi Ramamoorthi, Pat Hanrahan, "An efficient representation for irradiance environment maps", in SIGGRAPH, pp. 497-500, 2001.

[5] Radu Bogdan Rusu , Zoltan Csaba Marton , Nico Blodow , Mihai Dolha , Michael Beetz, "Towards 3D Point Cloud Based Object Maps for Household Environments," in Robotics and Autonomous Systems Journal, Volume 56 Issue 11, pp. 927-941 2008.

[6] Stefan Holzer, Radu Bogdan Rusu, M. Dixon, Suat Gedikli, and Nassir Navab, "Adaptive neighborhood selection for real-time surface normal estimation from organized point cloud data using integral images", in IROS, pp. 2684-2689, 2012.

[7] Derek Nowrouzezahrai, Patricio Simari, Eugene Fiume, "Sparse Zonal Harmonic Factorization for Efficient SH Rotation", in ACM Transactions on Graphic, 31(3): 23, 2012.
[8] Jadran Sirotkovic, Hrvoje Dujmic, Vladan Papic, "K-Means Image Segmentation on Massively Parallel GPU Architecture", in MIPRO, pp. 489-494, 2012.

[9] Berkin Bilgic, Berthold K. P. Horn, Ichiro Masaki, "Efficient Integral Image Computation on the GPU", in IEEE Intelligent Vehicles Symposium, pp. 528-533, 2010.

[10] Paul Debevec, "Rendering synthetic objects into real scenes: bridging traditional and image-ased graphics with global illumination and high dynamic range photography", in SIGGRAPH, pp. 189-198, 1998.

[11] Li Chen, Hui Lin, Shutao Li, "Depth image enhancement for Kinect using region growing and bilateral filter", in ICPR, pp. 3070-3073, 2012.

[12] Carlo Tomasi, Roberto Manduchi, "Bilateral filtering for gray and color images", in ICCV, pp. 839-846, 1998. 